\title{
Lazy zone bone remodeling theory and its relation to topology optimization
}

\author{
Anders Klarbring and Bo Torstenfelt
}

\section{Linköping University Post Print}

N.B.: When citing this work, cite the original article.

The original publication is available at www.springerlink.com:

Anders Klarbring and Bo Torstenfelt, Lazy zone bone remodeling theory and its relation to topology optimization, 2012, Annals of Solid and Structural Mechanics, (4), 1-2, 25-32. http://dx.doi.org/10.1007/s12356-012-0030-3

Copyright: Springer Verlag (Germany) http://www.springerlink.com/

Postprint available at: Linköping University Electronic Press http://urn.kb.se/resolve?urn=urn:nbn:se:liu:diva-85616 


\title{
Lazy zone bone remodeling theory and its relation to topology optimization
}

\author{
Anders Klarbring and Bo Torstenfelt \\ Mechanics, Department of Management and Engineering \\ The Institute of Technology, Linköping University \\ SE-581 83 Linköping, Sweden \\ anders.klarbring@liu.se, bo.torstenfelt@liu.se
}

\begin{abstract}
The connection between apparent density-type bone remodeling theories and density formulations of topology optimization is well known from a large number of publications and its theoretical basis has recently been discussed by making use of a dynamical systems approach to optimization. The present paper takes this connection one step further by showing how the Coleman-Noll procedure of rational thermodynamics can be used to derive general dynamical systems, where a special case includes the lazy zone concept of bone remodeling theory. It is also shown how a numerical solution method for the dynamical system can be developed by using the sequential convex approximation idea. The method is employed to obtain a series of solutions that show the influence of modeling parameters representing elements of plasticity and viscosity in the growth process.
\end{abstract}

\section{Introduction}

It is well known that there is a resemblance between, on the one hand, bone remodeling theories based on adaptive elasticity and apparent density, see Cowin and Hegedus [5], and, on the other hand, material distribution optimization problems such as that given by the SIMP formulation of topology optimization, see, e.g., Christensen and Klarbring [4]. Research aimed at applying topology optimization theory to bone remodeling can be found in, e.g., recent work by Jang and $\operatorname{Kim}[11,12,13]$, while studies with the converse aim, i.e., applying bone remodeling theories in structural and topology optimization, can be found in Penninger et al. [19] and Andreaus et al. [1]. The theoretical basis for this connection of theories is independently discussed in Jang et al. [14] and Klarbring and Torstenfelt $[15,16]$. To be more specific, in the latter works we showed that there is a one-to-one correspondence between a dynamical systems approach to SIMP topology optimization and the apparent density bone remodeling theory of Harrigan and Hamilton [8, 9]. The latter theory is based on a formal finite element formulation where Young's modules is related to the density by a power law, and a strain energy driven evolution equation for the density is used.

In the following we extend this analogy and develop a refined theory comprising the so-called lazy zone concept, meaning that, for a certain intermediate 
range of remodeling stimuli, no bone remodeling takes place. This concept was originally suggested in an experimental context by Carter [3] and first used in a computational model by Huiskes et al. [10]. It has since then been discussed and used in a large number of publications. Its general idea is depicted in Figure 1 of this paper where the apparent density evolution is represented by the $y$-axis and the strain energy stimuli is represented by the $x$-axis.

Our theoretical development starts from a Lyaponov-type inequality, which in the dynamical systems approach replaces the optimization formulation as the basic problem statement. We then observe that this inequality resembles the well-known dissipation inequality of thermodynamics, stated for instance in Gurtin et al. [7] or Frémond [6]. In fact, the logical status of these two inequalities are the same: we are to find a dynamical system that satisfies the inequality for all possible evolutions. In thermodynamics, this particular modeling step is known as the Coleman-Noll procedure, $[7,6]$. Following this procedure we derive a general dynamical system that when specialized takes the form of a lazy zone bone remodeling theory.

The resulting dynamical system is subsequently investigated by developing a numerical method based on an implicit time discretization, followed by a sequential explicit convex approximation in the standard manner of structural optimization, see, e.g., Christensen and Klarbring [4]. Next, we present a series of numerical solutions showing the influence of different modeling parameters. Finally, the conclusions of our investigations are summarized.

\section{Basic theory}

The standard formulation of structural optimization (the sizing, density or SIMP topology optimization version), see e.g. [4], is the following:

$$
\min _{\boldsymbol{u}, \boldsymbol{\rho} \in K} f(\boldsymbol{u}, \boldsymbol{\rho}) \quad \text { subject to } \boldsymbol{F}=\boldsymbol{K}(\boldsymbol{\rho}) \boldsymbol{u},
$$

where

- $\boldsymbol{\rho}=\left(\rho_{1}, \ldots, \rho_{E}\right)^{T}$ is a vector of $E$ design or configurational variables (see discussion below);

- $K$ is a set of such variables. In the following it takes the form of simple box constraints, i.e.,

$$
K=\prod_{i=1}^{E} K_{i}, \quad K_{i}=\left\{\rho_{i} \mid \underline{\rho} \leq \rho_{i} \leq \bar{\rho}\right\},
$$

where $\underline{\rho}$ and $\bar{\rho}$ are upper and lower bounds;

- the equilibrium equation of discrete structures

$$
\boldsymbol{F}=\boldsymbol{K}(\boldsymbol{\rho}) \boldsymbol{u},
$$

consists of a vector $\boldsymbol{F}$ of external forces, a displacement vector $\boldsymbol{u}$ and a positive semi-definite stiffness matrix $\boldsymbol{K}(\boldsymbol{\rho})$ that depends on $\boldsymbol{\rho}$;

- and $f(\boldsymbol{u}, \boldsymbol{\rho})$ is a goal or objective function. 
Here $E$ is the number of finite elements in the structure, and for simplicity we assume that there is one design variable for each such element.

In the SIMP formulation, the dependence of the stiffness matrix on the vector $\boldsymbol{\rho}$ is given by

$$
\boldsymbol{K}(\boldsymbol{\rho})=\sum_{i=1}^{E} \rho_{i}^{q} \boldsymbol{K}_{i}
$$

where $q$ is a positive exponent and $\boldsymbol{K}_{i}$ is an element stiffness matrix for a unit value of the design variable $\rho_{i}$. In topology optimization, $\boldsymbol{\rho}$ is a design variable whose elements take values between 0 and 1, i.e., $\rho=0$ and $\bar{\rho}=1$, and intermediate values are penalized by letting the exponent $q$ of (3) be larger than one in. In the bone remodeling interpretation of the same formulation, $\rho$ represents an apparent density, and while its lower bound is still zero, its upper bound corresponds to the density of compact bone. In the following we will refer to $\boldsymbol{\rho}$ as a vector of configurational variables, somehow including two different interpretations of the theory.

In this paper we use a dynamical systems approach and do not deal directly with the optimization problem (1). Rather, we regard the displacements and the configurational variables as depending on a time variable $t$, i.e., $\boldsymbol{u}=\boldsymbol{u}(t)$ and $\boldsymbol{\rho}=\boldsymbol{\rho}(t)$, and derive dynamical systems that satisfy certain Lyaponov-type inequalities. A simple such inequality, see [16], which can be applied when the external force $\boldsymbol{F}$ does not depend on time, reads

$$
\frac{d}{d t} f(\boldsymbol{u}, \boldsymbol{\rho}) \leq 0 \quad \text { for all } \boldsymbol{u} \text { and } \boldsymbol{\rho} \in K \text { such that } \boldsymbol{F}=\boldsymbol{K}(\boldsymbol{\rho}) \boldsymbol{u} .
$$

That is, we assume a quasi-static time evolution of the system such that the goal or objective function is never increasing. For biological systems, such as those involved in bone remodeling, stating an inequality like (4) implies that we have, experimentally or otherwise, observed an extremum property that we use as a postulate in modeling. The observation could be, e.g., that cost of material and/or mechanical efficiency are developing towards lower or homeostatic values during a growth process.

Obviously, (4) is a statement of different kind from (1): the latter problem is to be solved for a certain optimum $\left(\boldsymbol{u}^{*}, \boldsymbol{\rho}^{*}\right)$, while (4) is a requirement that should be satisfied by a dynamical system. An example of a dynamical system that satisfies (4) is

$$
\dot{\rho}_{i}=-\frac{\partial \tilde{f}(\boldsymbol{\rho})}{\partial \rho_{i}},
$$

where a superposed dot indicates a time derivative, and $\tilde{f}(\boldsymbol{\rho})=f(\boldsymbol{u}(\boldsymbol{\rho}), \boldsymbol{\rho})$, where $\boldsymbol{u}(\boldsymbol{\rho})$ solves (2), assuming that the stiffness matrix is non-singular. Equation (5) and the optimization problem (1) are now related in the sense that an equilibrium point $\boldsymbol{\rho}^{*}$ of (5) (satisfying $\partial \tilde{f}\left(\boldsymbol{\rho}^{*}\right) / \partial \rho_{i}=0$ ) has the property that $\left(\boldsymbol{u}\left(\boldsymbol{\rho}^{*}\right), \boldsymbol{\rho}^{*}\right)$ is a stationary point of (1) and the time sequence $\boldsymbol{\rho}=\boldsymbol{\rho}(t)$ defined by (5) converges towards such a point. However, not all dynamical systems that satisfy (4) define sequences that converge to stationary points of (1), and such systems will in fact be central to the present investigation. In such cases, however, we regard (4) as a statement that is closer to the physical behavior that we want to model than (1) is. 
We may also note that the case of a time-dependent load and objective function, as well as the extension to cyclic loading, is discussed in Klarbring and Torstenfelt [16].

\subsection{Dynamical system}

We will now derive a general dynamical system that satisfies the Lyaponov inequality (4). Such a derivation is analogous to that of the Coleman-Noll procedure of thermodynamics. As a first step we relax the equilibrium equation (2). Taking its time derivative (for constant $\boldsymbol{F}$ ) yields

$$
\sum_{i=1}^{E} \frac{\partial \boldsymbol{K}(\boldsymbol{\rho})}{\partial \rho_{i}} \boldsymbol{u} \dot{\rho}_{i}+\boldsymbol{K}(\boldsymbol{\rho}) \dot{\boldsymbol{u}}=\mathbf{0} .
$$

By multiplying this equation by a Lagrangian multiplier vector $\boldsymbol{\lambda}$ and adding to the inequality in (4), we get

$$
\left[\nabla_{u} f(\boldsymbol{u}, \boldsymbol{\rho})^{T}+\boldsymbol{\lambda}^{T} \boldsymbol{K}(\boldsymbol{\rho})\right] \dot{\boldsymbol{u}}+\sum_{i=1}^{E}\left[\frac{\partial f(\boldsymbol{u}, \boldsymbol{\rho})}{\partial \rho_{i}}+\boldsymbol{\lambda}^{T} \frac{\partial \boldsymbol{K}(\boldsymbol{\rho})}{\partial \rho_{i}} \boldsymbol{u}\right] \dot{\rho}_{i} \leq 0
$$

which must be satisfied for all evolutions of $\boldsymbol{u}, \boldsymbol{\lambda}$ and $\boldsymbol{\rho} \in K$. This requirement can be met by assuming the adjoint system

$$
\nabla_{u} f(\boldsymbol{u}, \boldsymbol{\rho})+\boldsymbol{K}(\boldsymbol{\rho}) \boldsymbol{\lambda}=\mathbf{0},
$$

where we have used the symmetry of $\boldsymbol{K}(\boldsymbol{\rho})$, and the inequality

$$
\sum_{i=1}^{E} R_{i} \dot{\rho}_{i} \geq 0
$$

where $R_{i}$ are configurational forces associated with growth that satisfy

$$
-R_{i} \in \frac{\partial f(\boldsymbol{u}, \boldsymbol{\rho})}{\partial \rho_{i}}+\boldsymbol{\lambda}^{T} \frac{\partial \boldsymbol{K}(\boldsymbol{\rho})}{\partial \rho_{i}} \boldsymbol{u}+\partial I_{K_{i}}\left(\rho_{i}\right) .
$$

In the last term of (8), the symbol $\partial$ indicates the subdifferential of a convex function, see, e.g., Rockafellar [20] or, for its use in mechanics, Panagiotopoulos [18] and Frémond [6]. The convex function is in this case the indicator function of the convex set $K_{i}$, i.e., a function that takes the value 0 for points inside the set and infinity for points outside the set.

A direct way of satisfying (7) for all $\dot{\rho}_{i}$ is to postulate the relation $R_{i}=$ $k_{i} \dot{\rho}_{i}$ for a non-negative function $k_{i}$ that could, e.g., depend on $\boldsymbol{\rho}, \boldsymbol{u}$ and their time derivatives. This was the route taken in Klarbring and Torstenfelt [16]. However, here we will take an alternative approach that will eventually lead to the incorporation of the lazy zone concept into the model. We will use positive convex potentials $D_{i}\left(\dot{\rho}_{i}\right)$, with the properties $0=D_{i}(0), 0 \in \partial D_{i}(0)$, and such that

$$
R_{i} \in \partial D_{i}\left(\dot{\rho}_{i}\right),
$$

see, e.g., Frémond [6]. The potential could depend on other variables than $\dot{\rho}_{i}$ in the same way as for $k_{i}$, but these act as parameters and the subdifferential is 
with respect to $\dot{\rho}_{i}$. Note that (9) can be multi-valued and the potential could take the value $\infty$, implying a constraint on admissible values of $\dot{\rho}_{i}$, which are features not present in $R_{i}=k_{i} \dot{\rho}_{i}$. On the other hand, $R_{i}=k_{i} \dot{\rho}_{i}$ could represent a nonmonotone relation between $R_{i}$ and $\dot{\rho}_{i}$, which is not included in (9) since $D$ needs to be convex. A non-convex generalization of (9) has, however, been suggested by Panagiotopoulos [18]. Using (9), the evolution law for configurational variables now reads

$$
0 \in \frac{\partial f(\boldsymbol{u}, \boldsymbol{\rho})}{\partial \rho_{i}}+\boldsymbol{\lambda}^{T} \frac{\partial \boldsymbol{K}(\boldsymbol{\rho})}{\partial \rho_{i}} \boldsymbol{u}+\partial D_{i}\left(\dot{\rho}_{i}\right)+\partial I_{K_{i}}\left(\rho_{i}\right) .
$$

In the following we will investigate the particular convex potential

$$
D_{i}=\frac{a_{i} c}{2} \dot{\rho}_{i}^{2}+\left\{\begin{aligned}
a_{i} d_{+} \dot{\rho}_{i} & \text { if } \dot{\rho}_{i} \geq 0 \\
-a_{i} d_{-} \dot{\rho}_{i} & \text { if } \dot{\rho}_{i}<0
\end{aligned}\right.
$$

where $c, d_{+}$and $d_{-}$are positive constants and $a_{i}$ may be interpreted as the volume (or area) of element $i$. The choice of this potential is basically one of convenience: it is the simplest potential that includes both a viscous growth effect, represented by the first term, and a plastic growth behavior, represented by the second term. However, an important motivation for choosing this particular potential is that it turns out to model the lazy zone behavior of bone remodeling, i.e., there is a range of stimulus for which no growth takes place, see $[3,10]$. With this potential (9) becomes

$$
R_{i} \in a_{i} c \dot{\rho}_{i}+ \begin{cases}a_{i} d_{+} & \text {if } \dot{\rho}_{i}>0 \\ a_{i}\left[-d_{-}, d_{+}\right] & \text {if } \dot{\rho}_{i}=0 \\ -a_{i} d_{-} & \text {if } \dot{\rho}_{i}<0\end{cases}
$$

Since this relation is multi-valued it is different from what can be represented by $R_{i}=k_{i} \dot{\rho}_{i}$, even when $k_{i}$ is a general function of $\dot{\rho}_{i}$.

\subsection{Special goal function}

The preceding presentation is valid for a general objective or goal function. Here we will specialize to a particular function that involves compliance (inverse of stiffness), $C$, and cost of material, $W$, defined by

$$
C=\frac{1}{2} \boldsymbol{F}^{T} \boldsymbol{u} \quad \text { and } \quad W=\sum_{i=1}^{E} a_{i} \rho_{i} .
$$

If the cost of material is measured by its weight, which is a natural choice, the constant $a_{i}$ would be the volume of element $i$. A natural goal function is now

$$
f=C+\mu W,
$$

for some constant $\mu$. For this special case, $\nabla_{u} f(\boldsymbol{u}, \boldsymbol{\rho})=\boldsymbol{F} / 2$, so by comparing the adjoint system (6) and the equilibrium equation (2) one finds that $\boldsymbol{\lambda}=$ $-\boldsymbol{u} / 2$. Moreover, assuming that $\boldsymbol{K}(\boldsymbol{\rho})$ is non-singular for all $\boldsymbol{\rho} \in K, \boldsymbol{u}$ can be seen as a function of $\boldsymbol{\rho}$, defined by (2). This implies that (10) takes the form

$$
0 \in \mu a_{i}-e_{i}(\boldsymbol{\rho})+\partial D_{i}\left(\dot{\rho}_{i}\right)+\partial I_{K_{i}}\left(\rho_{i}\right),
$$


where we have used the notation

$$
e_{i}(\boldsymbol{\rho})=\frac{1}{2} \boldsymbol{u}^{T} \frac{\partial \boldsymbol{K}(\boldsymbol{\rho})}{\partial \rho_{i}} \boldsymbol{u}
$$

Specializing further by taking the particular form of the function $D_{i}$ in (11), (13) becomes

$$
0 \in \mu a_{i}-e_{i}(\boldsymbol{\rho})+a_{i} c \dot{\rho}_{i}+ \begin{cases}a_{i} d_{+} & \text {if } \dot{\rho}_{i}>0 \\ a_{i}\left[-d_{-}, d_{+}\right] & \text {if } \dot{\rho}_{i}=0 \\ -a_{i} d_{-} & \text {if } \dot{\rho}_{i}<0 .\end{cases}
$$

When $c \neq 0$, this inclusion can be inverted and we obtain

$$
\dot{\rho}_{i}=\left\{\begin{array}{ccc}
c^{-1}\left(\frac{e_{i}(\boldsymbol{\rho})}{a_{i}}-\mu-d_{+}\right) & \text {if } & \frac{e_{i}(\boldsymbol{\rho})}{a_{i}}>\mu+d_{+} \\
0 & \text { if } & \mu-d_{-} \leq \frac{e_{i}(\boldsymbol{\rho})}{a_{i}} \leq \mu+d_{+} \\
c^{-1}\left(\frac{e_{i}(\boldsymbol{\rho})}{a_{i}}-\mu+d_{-}\right) & \text {if } & \frac{e_{i}(\boldsymbol{\rho})}{a_{i}}<\mu-d_{-} .
\end{array}\right.
$$

Note that since $e_{i}(\boldsymbol{\rho}) \geq 0$, due to positive semi-definiteness of $\boldsymbol{K}_{i}$, we need to have $\mu>d_{-}$for the last line to ever be applicable. This means that bone resorption never takes place unless this condition holds, and the physical interpretation is that the cost of bone has to be higher than a measure of energy consumption when removing bone. Equation (15) appears as a purely phenomenological equation in the bone remodeling literature. It is thought of as modeling the so called lazy zone behavior, which means that for mature bone there is a range of stimuli for which the bone is stable and does not remodel. Note that the stimuli turns out to be

$$
\frac{e_{i}(\boldsymbol{\rho})}{a_{i}}=q U_{i}, \quad U_{i}=\frac{1}{2} \frac{\boldsymbol{u}^{T} \rho_{i}^{q} \boldsymbol{K}_{i} \boldsymbol{u}}{a_{i} \rho_{i}},
$$

where $U_{i}$ is the mass specific strain energy of element $i$. Equation (15) is illustrated in Figure 1.

When $c=0,(14)$ represents a purely plastic behavior; it implies

$$
\mu-d_{-} \leq \frac{e_{i}(\boldsymbol{\rho})}{a_{i}} \leq \mu+d_{+},
$$

together with the growth direction criterion

$$
\dot{\rho}_{i}\left\{\begin{array}{cccc}
\geq 0 & \text { if } & \frac{e_{i}(\boldsymbol{\rho})}{a_{i}}=\mu+d_{+} \\
=0 & \text { if } & \mu-d_{-}<\frac{e_{i}(\boldsymbol{\rho})}{a_{i}}<\mu+d_{+} \\
\leq 0 & \text { if } & \frac{e_{i}(\boldsymbol{\rho})}{a_{i}}=\mu-d_{-} .
\end{array}\right.
$$

\section{$3 \quad$ Numerical method}

For the numerical integration of (13), including (14) as a special case, we discretize time into steps of length $\Delta t$. Given a solution $\rho^{n}$ at time $t_{n}$, we find the 


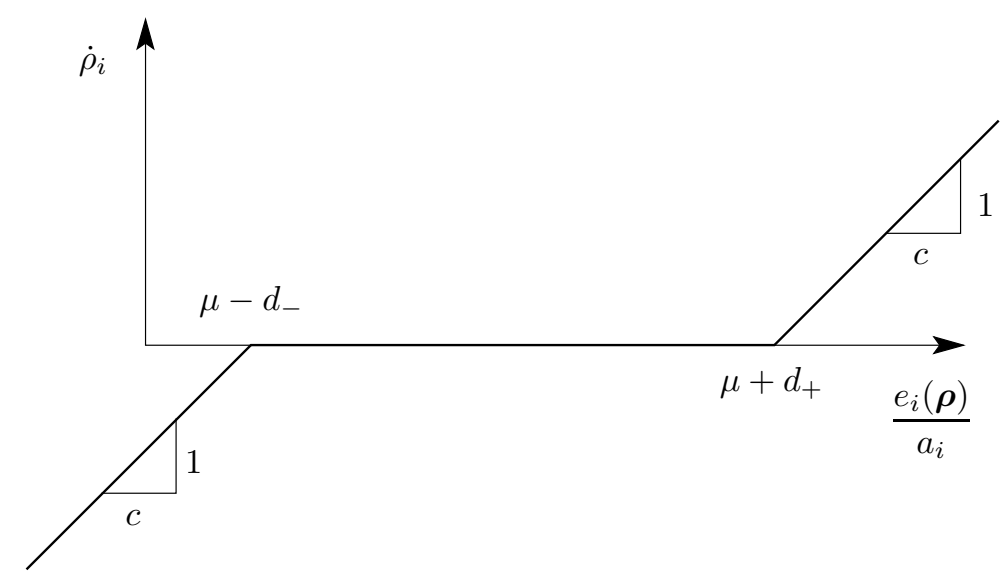

Figure 1: Geometric representation of equation (15), including a lazy zone of length $d_{-}+d_{+}$.

solution $\rho$ at time $t_{n+1}$ by solving

$$
0 \in \frac{d f(\boldsymbol{u}(\boldsymbol{\rho}), \boldsymbol{\rho})}{d \rho_{i}}+\partial I_{K_{i}}\left(\rho_{i}\right)+\partial D_{i}\left(\frac{\rho_{i}-\rho_{i}^{n}}{\Delta t}\right) .
$$

This is essentially an implicit time discretization scheme and (16) defines a unilateral stationary point of the optimization problem

$$
(\mathcal{M})^{n} \min _{\boldsymbol{\rho} \in K}\left[f(\boldsymbol{u}(\boldsymbol{\rho}), \boldsymbol{\rho})+\Delta t \sum_{i=1}^{E} D_{i}\left(\frac{\rho_{i}-\rho_{i}^{n}}{\Delta t}\right)\right] .
$$

To solve $(\mathcal{M})^{n}$, in the case when $D_{i}$ is given by (11), we suggest a sequential convex approximation strategy, see Christensen and Klarbring [4]. This means solving an iterative sequence of problems defined by convex approximations of the objective function of $(\mathcal{M})^{n}$. These approximations should involve explicit functions of $\boldsymbol{\rho}$, as opposed to the original objective function where $\boldsymbol{u}(\boldsymbol{\rho})$ is defined implicitly by solving (2). The first term of the goal function, see (12), is linearized in the, so-called, intervening variables $\rho_{i}^{-\alpha}, \alpha>0$, see Christensen and Klarbring [4]. If the linearization is made at the point $\boldsymbol{\rho}^{k}$ we obtain

$$
f(\boldsymbol{u}(\boldsymbol{\rho}), \boldsymbol{\rho}) \approx \text { const. }+\sum_{i=1}^{E}\left[e_{i}\left(\boldsymbol{\rho}^{k}\right) \alpha^{-1}\left(\rho_{i}^{k}\right)^{1+\alpha} \rho_{i}^{-\alpha}+\mu a_{i} \rho_{i}\right] .
$$

The approximation of $(\mathcal{M})^{n}$ then reads

$$
(\mathcal{M})^{n, k} \quad \min _{\boldsymbol{\rho} \in K} \sum_{i=1}^{E} \varphi_{i}^{n, k}\left(\rho_{i}\right)=\sum_{i=1}^{E} \min _{\rho_{i} \in K_{i}} \varphi_{i}^{n, k}\left(\rho_{i}\right),
$$

where

$$
\begin{aligned}
\varphi_{i}^{n, k}\left(\rho_{i}\right)=e_{i}\left(\boldsymbol{\rho}^{k}\right) \alpha^{-1}\left(\rho_{i}^{k}\right)^{1+\alpha} \rho_{i}^{-\alpha}+\mu a_{i} \rho_{i} \\
+\frac{1}{2} c_{i} a_{i} \frac{\left(\rho_{i}-\rho_{i}^{n}\right)^{2}}{\Delta t}+\left\{\begin{aligned}
d_{+} a_{i}\left(\rho_{i}-\rho_{i}^{n}\right) & \text { if } \rho_{i} \geq \rho_{i}^{n} \\
-d_{-} a_{i}\left(\rho_{i}-\rho_{i}^{n}\right) & \text { if } \rho_{i}<\rho_{i}^{n} .
\end{aligned}\right.
\end{aligned}
$$


The switch of min and sum in $(\mathcal{M})^{n, k}$ is possible due to the separability of the approximation (17). The solution of the inner convex subproblem of minimizing $\varphi_{i}^{n, k}\left(\rho_{i}\right)$ over the interval $K_{i}$ will be one out of five types: the minimum is taken

(i) at the left endpoint $\rho_{i}=\underline{\rho}$,

(ii) at the right endpoint $\rho_{i}=\bar{\rho}$,

(iii) at the non-differentiable point $\rho_{i}=\rho_{i}^{n}$,

(iv) at a stationary point on the interval $\underline{\rho}<\rho_{i}<\rho_{i}^{n}$, or

(iv) at a stationary point on the interval $\rho_{i}^{n}<\rho_{i}<\bar{\rho}$.

Making the trail assumption that a stationary point occurs in the interval $\rho_{i}^{n}<$ $\rho_{i}<\bar{\rho}$ we find

$$
\frac{\partial \varphi_{i}^{n, k}\left(\rho_{i}\right)}{\partial \rho_{i}}=a_{i}\left(\mu+d_{+}\right)+\frac{c_{i} a_{i}}{\Delta t}\left(\rho_{i}-\rho_{i}^{n}\right)-e_{i}\left(\boldsymbol{\rho}^{k}\right)\left(\rho_{i}^{k}\right)^{1+\alpha} \rho_{i}^{-(1+\alpha)}=0 .
$$

For general constants, this is an equation that is not explicitly solvable. Nonetheless, we call its unique solution ${ }^{1} \hat{\rho}_{i}^{k}$ and for the special case $c_{i}=0$ we have

$$
\rho_{i}=\left(\frac{e_{i}\left(\boldsymbol{\rho}^{k}\right)}{a_{i}\left(\mu+d_{+}\right)}\right)^{\frac{1}{1+\alpha}} \rho_{i}^{k}
$$

Assuming that a stationary point is taken in $\rho<\rho_{i}<\rho_{i}^{n}$ we find the same equation where $d_{+}$is replaced for $-d_{-}$. The corresponding solution is denoted ${ }^{2} \hat{\rho}_{i}^{k}$, and we end up with the following updating alternatives:

$$
\begin{array}{cccc}
\text { if } & { }^{1} \hat{\rho}_{i}^{k} \geq \bar{\rho} & \text { then } & \rho_{i}^{k+1}=\bar{\rho} \\
\text { if } & { }^{2} \hat{\rho}_{i}^{k} \leq \underline{\rho} & \text { then } & \rho_{i}^{k+1}=\underline{\rho} \\
\text { if } & \bar{\rho}>{ }^{1} \hat{\rho}_{i}^{k}>\rho_{i}^{n} & \text { then } & \rho_{i}^{k+1}={ }^{1} \hat{\rho}_{i}^{k} \\
\text { if } & \underline{\rho}<{ }^{2} \hat{\rho}_{i}^{k}<\rho_{i}^{n} & \text { then } \quad \rho_{i}^{k+1}={ }^{2} \hat{\rho}_{i}^{k} \\
\text { if } & { }^{2} \hat{\rho}_{i}^{k} \geq \rho_{i}^{n} \geq{ }^{1} \hat{\rho}_{i}^{k} & \text { then } & \rho_{i}^{k+1}=\rho_{i}^{n} .
\end{array}
$$

Finally it should be remarked that when the exponent $q$ in (3) is grater than one, it is well known that the underlying continuum problem may lack a solution, which shows up in the discrete problem as checkerboards and mesh dependence, see [4]. To avoid such incorrect solutions, we use the filtering scheme suggested by Sigmund, discussed in [21]. It essentially means using a weighted averaging of $e_{i}\left(\boldsymbol{\rho}^{k}\right)$ over nearby elements. It is interesting that checkerboards appeared also in early bone remodeling calculations, and to remedy this Mullender et al. [17] introduced a spatial influence function and the notion of sensor cells, in close analogy with the filter approach used in topology optimization.

\section{Numerical results}

We like to investigate the influence on the solution of the parameters $c$ (viscosity), $d_{+}$and $d_{-}$(plasticity), as well as the initial distribution of the configurational variable $\boldsymbol{\rho}$. The loading will be a step loading (time independent $\boldsymbol{F}$ ) and we will presents plots showing converged, i.e., equilibrium, distributions of 
$\boldsymbol{\rho}$. We divide the investigation into two parts: first the convex case $q=1$ and then the non-convex case of $q=3$ are treated. For a discussion of convexity and non-convexity of these goal functions we refer to Svanberg [22]. The dimensions of the computational domain are 1.8 by 0.3 and the load is uniformly distributed over a boundary segment of length 0.06. Due to symmetry, we model half of the domain. Poisson's ratio is taken to be 0.3. The finite element discretization is obtained by four node square Lagrangian displacement elements of length 0.01 resulting in a total of 2700 elements. The upper and lower bounds on the configurational variable are $\underline{\rho}=0.001 \approx 0$ and $\bar{\rho}=1$. For each set of problems, we fix the value of the constant $\mu$ by first solving a classical stiffness optimization problem with volume constraint. In such a problem $\mu$ corresponds to a Lagrangian multiplier, Christensen and Klarbring [4].

\subsection{Convex case}

In Figure 2 we show the influence of the plasticity parameter $d_{-}$, while $c$ and $d_{+}$are set to zero. In the the top plot, however, $d_{-}=0$, which reduces the problem to a classical problem (essentially the convex variable thickness sheet problem, see [4]), while in the other two plots $d_{-}$is set to a fraction of $\mu$, namely $0.5 \mu$ and $0.8 \mu$. Since $c=0$, the problem is reduced to a single time step: after convergence of the first time step there is no change in the solution in the following steps since the load is constant. This is a consequence of the time scale independence of plasticity problems. However, even though the scale is unimportant, the problem is path dependent when $d_{-} \neq 0$, implying that the initial distribution of $\boldsymbol{\rho}$ has an influence on the final solution. In Figure 2 it is seen that the plasticity effect prevents material from being removed: the top picture represents the minimum of the goal function $f=C+\mu W$ while in the other two pictures the plasticity or friction effect prevents the solution from reaching this minimum. If the displacement value for the central point under the load is normalized so that its value in the top picture of Figure 2 is 1, the corresponding displacements, in the same scale, for the other two solutions are 0.7976 and 0.6501 . These numbers indicate the obvious fact that stiffer structures are obtained when less material is removed.

In Figure 3 the opposite effect to that in Figure 2 is illustrated: the plasticity parameter $d_{+}$is varied, while $c$ and $d_{-}$are set to zero and we start from an initial configurational parameter which takes the lower value $\rho \approx 0$ in all elements. The upper plot is the same as the upper picture in Figure 2, i.e., it is the reference case of all three parameters being zero. In the lower two plots we have set $d_{+}$ equal to $\mu$ and $2 \mu$, respectively. In this case the plasticity or friction prevents material from being added and the displacement under the load becomes, when normalized, 1, 1.309 and 1.550 from top to bottom.

We now turn to the influence of the parameter $c$. In Figure 4 we start from a distribution $\rho_{i}=\bar{\rho}$ in all elements and $d_{-}=0.5 \mu$. We then show converged, i.e., equilibrium distributions, for three values of $c$, namely $0,10^{-14} \Delta t$ and $10^{-12} \Delta t$. It is clear that $c$ has the effect of smoothing out the solution, increasing the length scale of its geometrical features. The normalized displacements become $1,1.003$ and 1.010. Thus, a non-zero $c$ gives a distribution that represents a marginally less stiff structure. Note that viscosity (non-vanishing $c$ ) introduces a time scale into the problem and we have to use a sequence of time steps. A similar test on the influence of $c$ has also been done for the case with initial 

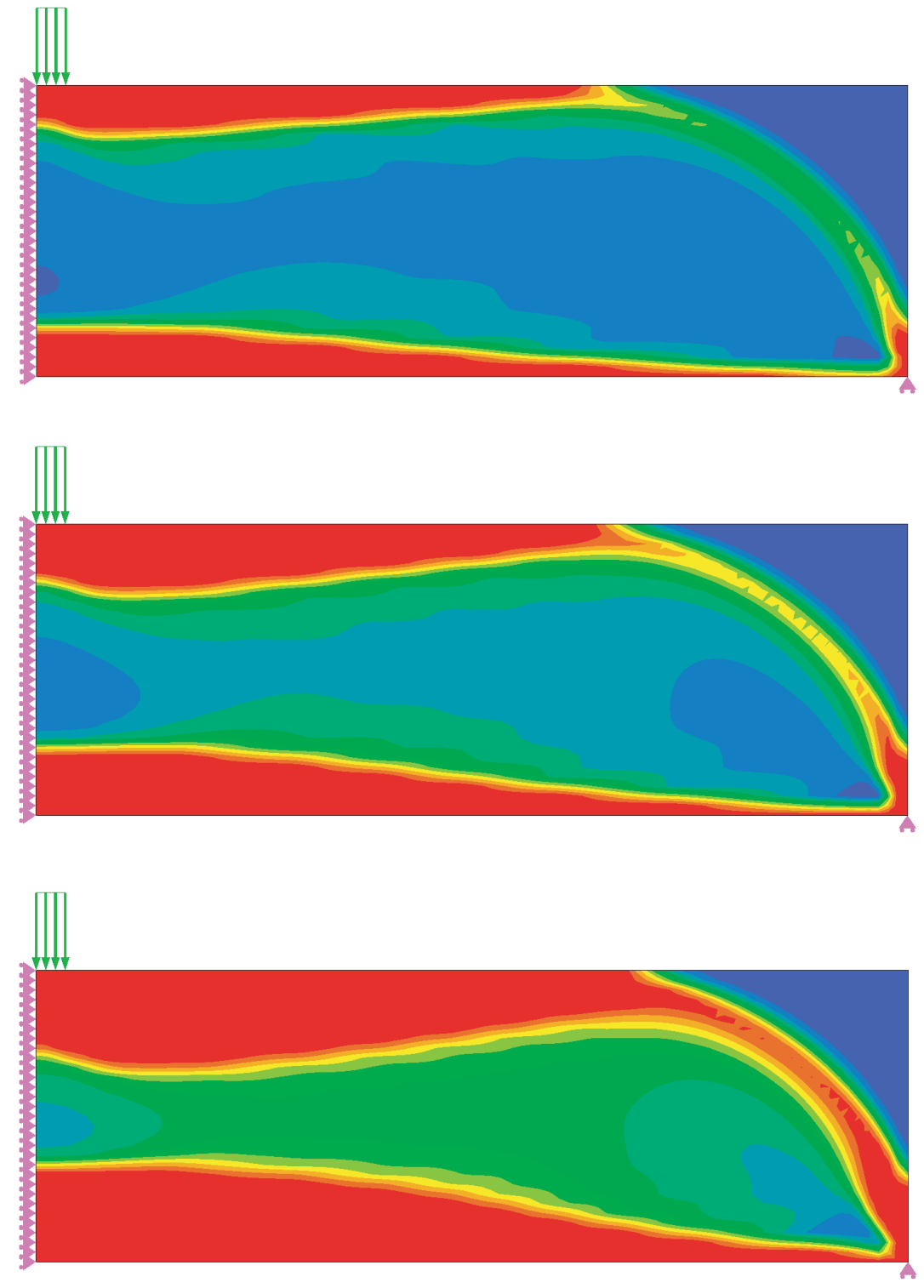

Figure 2: The influence of the plasticity parameter $d_{-}$when $c=d_{+}=0$, we start from an initial distribution of $\rho_{i}=\bar{\rho}$ for all elements, and $q=1$. From top to bottom we have set $d_{-}$equal to $0,0.5 \mu$ and $0.8 \mu$. 

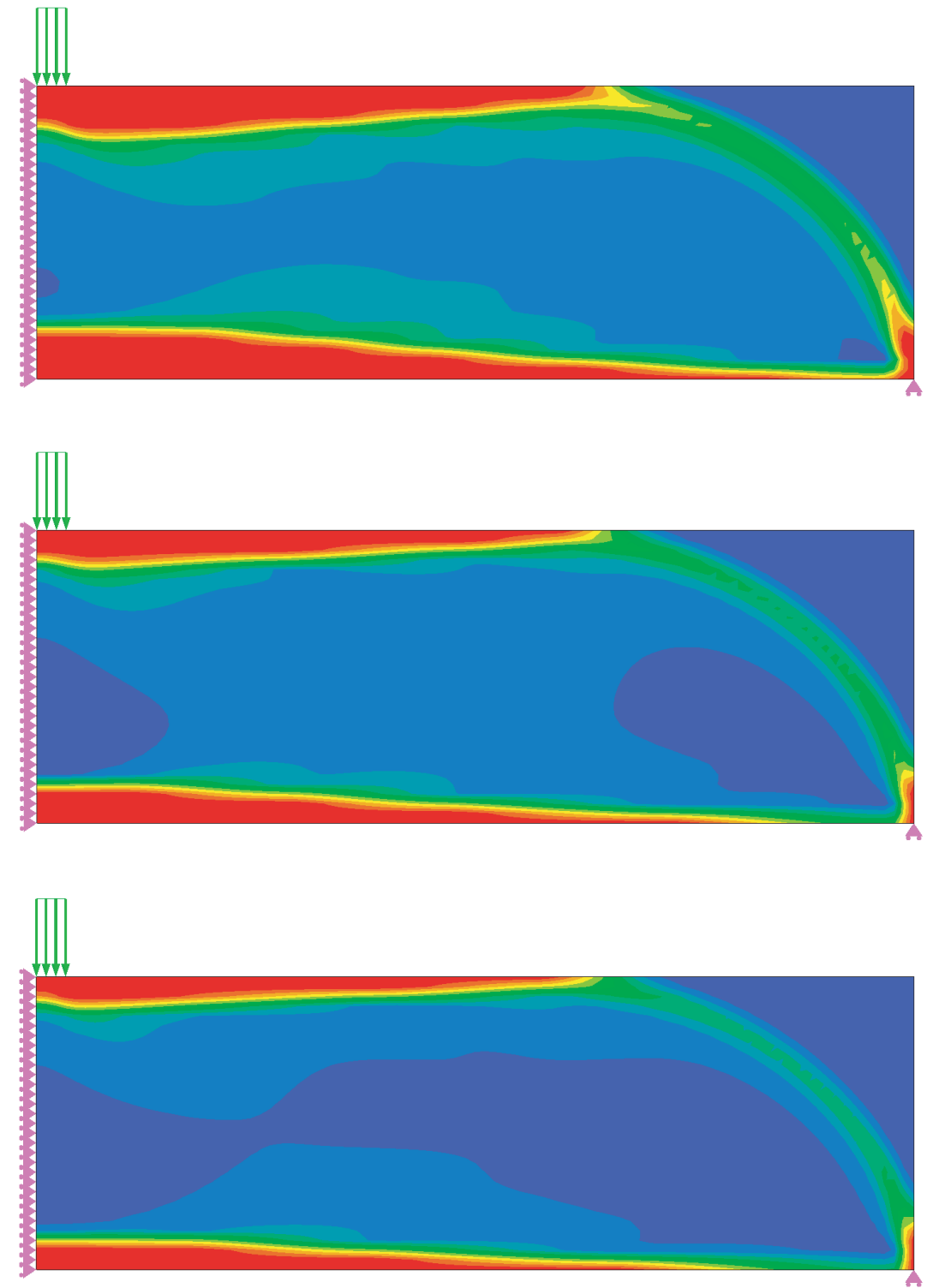

Figure 3: The influence of the plasticity parameter $d_{+}$when $c=d_{-}=0$, we start from an initial distribution of $\rho_{i}=\underline{\rho}$ for all elements, and $q=1$. From top to bottom we have set $d_{+}$equal to $0, \mu$ and $2 \mu$. 
distribution $\underline{\rho} \approx 0$. Essential the same behavior as in the reported test is found.
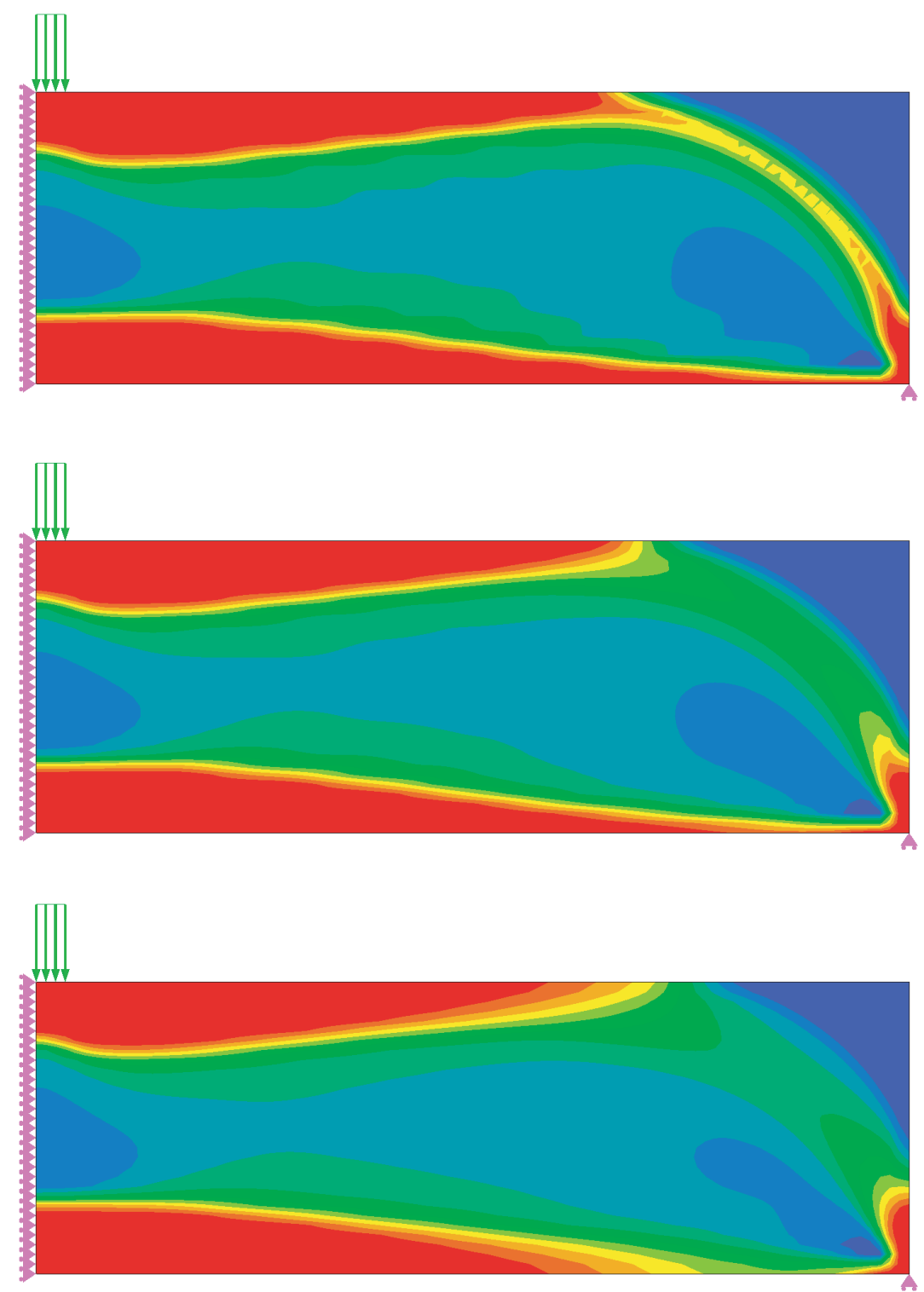

Figure 4: The influence of the viscosity parameter $c$ when $d_{+}=0, d_{-}=0.5 \mu$, we start from an initial distribution of $\rho_{i}=\bar{\rho}$ for all elements, and $q=1$. From top to bottom we have set $c$ equal to $0,10^{-14} \Delta t$ and $10^{-12} \Delta t$. 


\section{$4.2 \quad$ Non-convex case}

The sequence of test runs reported above can also be made for the non-convex case of $q=3$. The solutions then become more of the black-white type but are otherwise exhibiting a behavior similar to the convex case. In Figure 5 we start from $\rho_{i}=\bar{\rho}$ and test different values of $d_{-}$. The normalized displacements are from top to bottom $1,0.7562$ and 0.5478 . The similar problem when starting
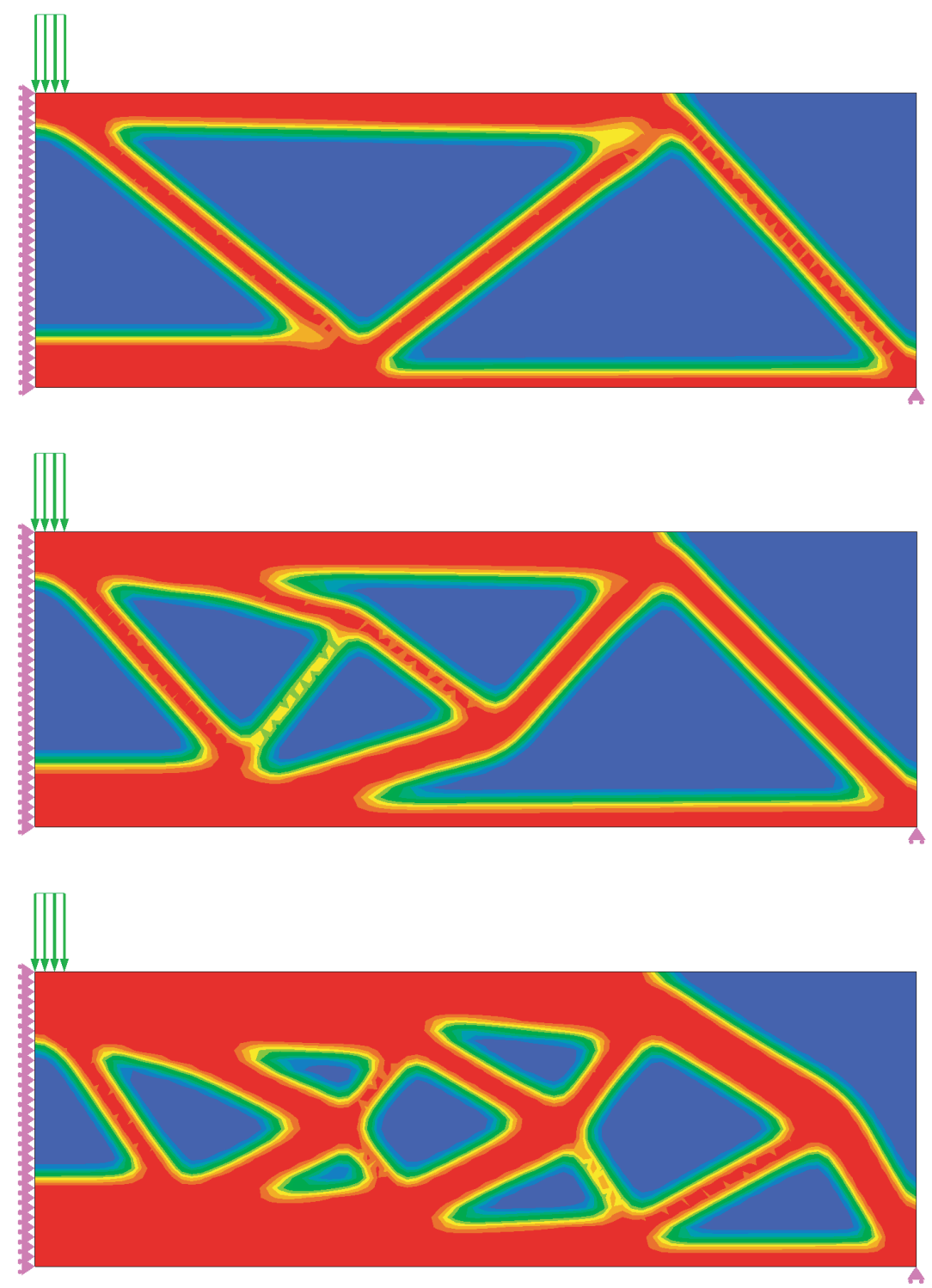

Figure 5: The influence of the plasticity parameter $d_{-}$when $c=d_{+}=0$, we start from an initial distribution of $\rho_{i}=\bar{\rho}$ for all elements, and $q=3$. From top to bottom we have set $d_{-}$equal to $0,0.5 \mu$ and $0.8 \mu$. 
from $\rho_{i}=\rho$ shows the expected behavior that a higher value of $d_{+}$produces a thinner structure but the topology is the same in all calculations.

Finally, we study the influence of the parameter $c$ in the non-convex case. We start again from a full distribution $\rho_{i}=\bar{\rho}$ and use the same values for $c$ as in the convex case. The normalized displacements from top to bottom become $1,1,004$ and 1,1586. The lower picture, representing the largest viscosity, shows quite a different behavior from the other solutions. Clearly, a sufficiently large viscosity smooths out the solution and does not give a black-white solution.

\section{Conclusions and discussion}

The connection between certain bone remodeling theories and a density approach to topology optimization, which was previously discussed in Klarbring and Torstenfelt [15, 16], has been taken one step further in the present paper, showing how more refined bone remodeling theories, e.g., involving a lazy zone, fits into a dynamical systems approach to optimization. The well-known Coleman-Noll procedure of rational thermodynamics turns out to be applicable in the derivation of dynamical systems. This is seen essentially by letting the objective function play the role of a thermodynamic free energy. The resulting modeling approach is flexible and by making particular choices for the objective function and the dissipation function particular remodeling theories can be obtained, one such theory is demonstrated to involve the lazy zone context. Moreover, it is shown that the lazy zone model is related to elements of plasticity and viscosity in the growth process.

Furthermore, we show how the general idea of linearizing in an intermediate variable, well known in structural optimization, can be used in obtaining an algorithm for the solution of the dynamical system. An extensive set of numerical tests indicate the effect of different parameters involved. In particularly, we find that inclusion of viscosity tends to smooth out an otherwise black and white solution.

The discussion and developments of this paper was limited to static loading, since our main goal was to show the inclusion of the lazy zone concept into our previously developed theory. However, in [16] we have shown how to use time dependent loads and a time dependent goal function. In fact, in that paper we also discussed the possibility of treating cyclic loading by means of several simultaneous load cases. Obviously, such cyclic loading, arising typically in walking, is important in bone remodeling calculations. Although, this theory of time dependent loading was presented in the context of the equation $R_{i}=k_{i} \dot{\rho}_{i}$, instead of (9), it is easily extended to this later case.

Finally, we note that although the problems discussed in this paper are obviously test problems, chosen to demonstrate the impact of different modeling parameters, the general approach is not limited to simple geometries in two dimensions. This has been amply demonstrated in the topology optimization literature and by the existence of several widely used commercial programs. In the context of bone remodeling, the recent paper by Boyle and Kim [2] shows that three-dimensional bone remodeling calculations for real geometries are possible. 

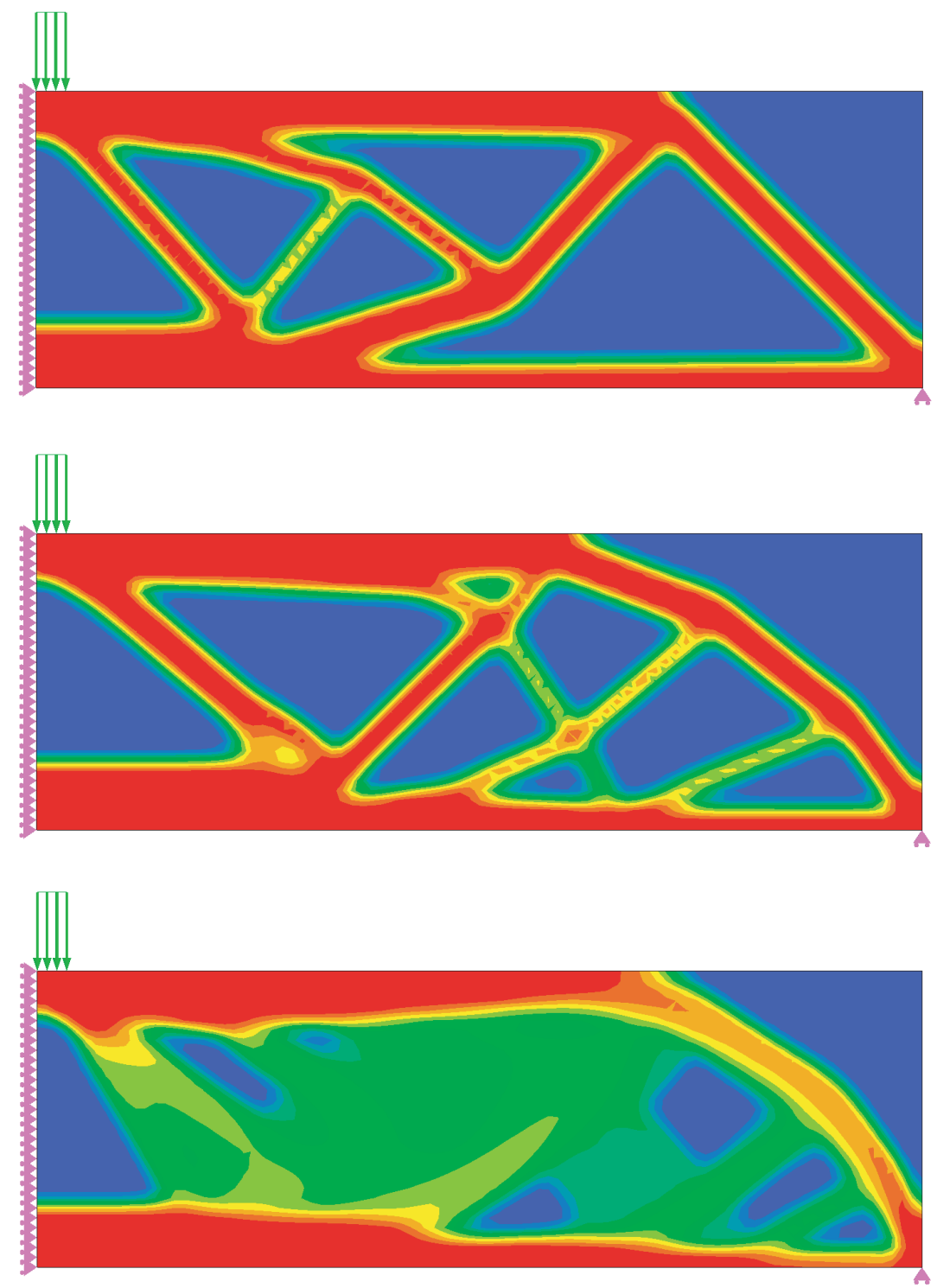

Figure 6: The influence of the viscosity parameter $c$ when $d_{+}=0, d_{-}=0.5 \mu$, we start from an initial distribution of $\rho_{i}=\bar{\rho}$ for all elements, and $q=3$. From top to bottom we have set $c$ equal to $0,10^{-14} \Delta t$ and $10^{-12} \Delta t$. 


\section{References}

[1] U. Andreaus, M. Colloca, D. Iacoviello and M. Pignataro, Optimal-tuning PID control of adaptive materials for structural efficiency, Structural Optimization 43 (2011) 43-59.

[2] C. Boyle and I.Y. Kim, Three-dimensional micro-level computational study of Wolffs law via trabecular bone remodeling in the human proximal femur using design space topology optimization, Journal of Biomechanics 44 (2011) 935942 .

[3] D.R. Carter DR (1984), Mechanical loading histories and cortical bone remodelling, Calcified Tissue International 36 (Suppl 1) (1984) S19S24.

[4] P.W. Christensen and A. Klarbring, An Introduction to Structural Optimization, Springer 2009.

[5] S.C. Cowin and D.H. Hegedus, Bone remodeling I: a theory of adaptive elasticity, Journal of Elasticity 6 (1976) 313-326.

[6] M. Frémond, Non-Smooth Thermomechanics, Springer, Berlin 2002.

[7] M.E. Gurtin, E. Fried and L. Anand, The Mechanics and Thermodynamics of Continua, Cambridge University Press, New York 2010.

[8] T.P. Harrigan and J.J. Hamilton, Necessary and sufficient conditions for global stability and uniqueness in finite element simulations of adaptive bone remodeling, International Journal of Solids and Structures 31(1) (1994) 97107.

[9] T.P. Harrigan and J.J. Hamilton, Bone remodeling and structural optimization, Journal of Biomechanics 27(3) (1994) 323-328.

[10] R. Huiskes, H. Weinans, H.J. Grootenboer, M. Dalstra, B. Fudala, T.J. Slooff, Adaptive bone-remodeling theory applied to prosthetic-design analysis, Journal of Biomechanics 20(11-12) (1987) 1135-1150.

[11] I.G. Jang and I.Y. Kim, Computational study of Wolff's law with trabecular architecture in the human proximal femur using topology optimization, Journal of Biomechanics 41(11) (2008) 2353-2361.

[12] I.G. Jang and I.Y. Kim, Computational simulation of trabecular adaptation progress in human proximal femur during growth, Journal of Biomechanics 42(5) (2009) 573-580.

[13] I.G. Jang and I.Y. Kim, Computational simulation of simultaneous cortical and trabecular bone change in human proximal femur during bone remodeling, Journal of Biomechanics 43(2) (2010) 294-301.

[14] I.G. Jang, I.Y. Kim and B.M. Kwak, Analogy of strain energy desnity based bonde-remodeling algorithm and structural topology optimization, Journal of Biomechanical Engineering 131 (2009) 011012-1-011012-7.

[15] A. Klarbring and B. Torstenfelt, Dynamical systems and topology optimization, Structural and Multidisciplinary Optimization, 42(2) (2010) 179-192. 
[16] A. Klarbring and B. Torstenfelt, Dynamical systems, SIMP, bone remodeling and time dependent loads, Structural and Multidisciplinary Optimization, 45 (2012) 359366,

[17] M.G. Mullender, R. Huiskes and H. Weinans, A physiological approach to the simulation of bone remodeling models, Journal of Biomechanics 27 (1994) 1489-1394.

[18] P.D. Panagiotopoulos, Inequality problems in mechanics and applications: convex and nonconvex functions, Birkhäuser 1985.

[19] C.L. Penninger, L.T. Watson, A. Tovar and J.E. Renaud, Convergence analysis of hybrid cellular automata for topology optimization, Structural Optimization 40 (2010) 271-282.

[20] R.T. Rockafellar, Convex Analysis, Princton University Press 1972.

[21] O. Sigmund, Morphology-based black and white filters for topology optimization, Structural and Multidisciplinary Optimization 33 (2007) 401-424.

[22] K. Svanberg, On the convexity and concavity of compliances, Structural Optimization 7(1-2) (1994) 42-46. 